\title{
MAGNETIC PROPERTIES OF ZINC DOPED FERRITES
}

\author{
M.I. ABD EL-ATi ${ }^{a}$, AzIZ M. KAFAFY ${ }^{b}$, AND A. TAWFIK ${ }^{a}$ \\ aPhysics Department, Faculty of Science, Tanta University, \\ Tanta, Egypt \\ ${ }^{b}$ Geology Department, Faculty of Science, Tanta University, \\ Tanta, Egypt
}

(Received November 5, 1990)

\begin{abstract}
A series of samples of the system $\mathrm{Co}_{1-x} \mathrm{Zn}_{x} \mathrm{Fe}_{2} \mathrm{O}_{4}(\mathrm{x}=0.0,0.3,0.4,0.5$ and 0.8 ) have been prepared by the usual ceramic technique. X-ray analysis shows that they are cubic spinel (single phase). The lattiçe parameter $a$ and the bulk density $D$ are measured for the samples. The substitution experiments replacing $\mathrm{Co}^{2+}$ by $\mathrm{Zn}^{2+}$ furnish new significant fact concerning the magnetic behaviour of ferrites. The magnetic strength of the magnetic ions on the $B-$ and $A$-sites can be varried relative to each other by the substitution of $\mathrm{Zn}^{2+}$. The increase of density of the composition increases the magnetic interaction of the dipole moment at the B-sites.
\end{abstract}

PACS numbers: 75.50.Dd, 75.50.Gg

\section{Introduction}

An insight into the magnetic behaviour of the ferrites can be gained by performing certain substitutions for the ferric ion. Michel and Pouillard [1] substituted $\mathrm{Al}^{3+}$ for $\mathrm{Fe}^{3+}$ in $\mathrm{Fe}_{3} \mathrm{O}_{4}$, and they found that both the Curie temperature and size of the unit cell decreased linearly with the amount of aluminium added until a point was reached where approximately one ion out of seven $\mathrm{Fe}^{3+}$ was replaced by one $\mathrm{Al}^{3+}$; beyond this point, both the Curie temperature and size of unit cell remained constant. They concluded from this work that only a limited amount of aluminium can be held in solid solution with $\mathrm{Fe}_{3} \mathrm{O}_{4}$.

Thermomagnetic investigations of promoted, unreduced iron oxide catalysts were carried out by Maxwell, Smart, and Brunauer [2] on $\mathrm{Fe}_{3} \mathrm{O}_{4}$ containing up to about 35 mole percent of $\mathrm{Al}_{2} \mathrm{O}_{3}$. The Curie temperature remained constant with 
a reduction in the intensity of magnetization roughly proportional to the volume occupied by the $\mathrm{Fe}_{3} \mathrm{O}_{4}$ which indicated that the $\mathrm{Al}_{2} \mathrm{O}_{3}$ existed either as a separate phase or possibly in combination with $\mathrm{FeO}$ to form a ferrous aluminate.

Guillaud and Michel [3] have found a linear decrease in the saturation magnetic moment for magnetite with aluminium substituted until there was approximately 0.20 of an aluminium ion per molecule, presumably in solid solution. Although the authors state that their data is in agreement with the Neel theory [4], the way in which this agreement is established is not clear because of the lack of sufficient information as to the distribution of the magnetic ions in the lattice.

The replacement of trivalent iron in $\mathrm{Fe}_{3} \mathrm{O}_{4}$ is complicated by the fact that the iron ion can go from the ferrous to ferric state rather easily by a simple transfer of an electron. It, therefore, is desirable to perform substitution experiments with a material having more stable divalent ion.

$\mathrm{Zn}^{2+}$ ions prefer to occupy tetrahedral sites in the spinel lattice and in the mixed $\mathrm{Co}-\mathrm{Zn}$ ferrites [5], the molecular formula is

$$
\mathrm{Zn}_{x}^{2+} \mathrm{Fe}^{3+}\left[\mathrm{Co}_{1-x}^{2+} \mathrm{Fe}^{3+}\right] \mathrm{O}_{4} \text {. }
$$

During sintering some of $\mathrm{Fe}^{3+}$ changes to $\mathrm{Fe}^{2+}$, the electronic conductivity occurs due to exchange of electrons between trivalent and divalent iron ions $\mathrm{Fe}^{3+}$ and $\mathrm{Fe}^{2+}$, respectively.

The aim of the present work is to study the effect of $\mathrm{Zn}$ additions on the lattice parameter, the density and the magnetic properties of $\mathrm{CoFe}_{2} \mathrm{O}_{4}$ ferrites.

\section{Experimental.}

\subsection{Sample preparation}

Samples of the system $\mathrm{Co}_{1-x} \mathrm{Zn}_{x} \mathrm{Fe}_{2} \mathrm{O}_{4}(x=0.0,0.3,0.4,0.5$ and 0.8$)$ were prepared using the usual ceramic technique. The pure oxides were mixed and then ground to a very fine powder using an agate mortar made of carborundum. The samples in form of discs were sintered at $1200^{\circ} \mathrm{C}$ for two hours and slowly cooled to room temperature. The samples were polished to have uniform parallel surfaces. For magnetic measurements contacts on the sample surface were made by silver paste.

\subsection{Lattice parameter and density measurements}

The X-ray diffraction pattern for each sample was recorded by using a Shimadzu X-ray diffractometer (Model X D-3). The powder specimens were exposed to $\mathrm{Cu} K_{\alpha}$ radiation. The lattice parameter $a$ was calculated from the X-ray diffraction pattern [5]. The bulk density $D$ is determined by using the Archimedes principle in toluene according to the following equation:

$$
D=\frac{W_{\mathrm{s}}}{W_{\mathrm{t}}} \rho_{\mathrm{t}}
$$


where $W_{\mathbf{s}}$ denotes the weight of the specimen in air, $W_{\mathrm{t}}$ the apparent weight loss in toluene, and $\rho_{\mathrm{t}}$ the density of toluene.

The magnetic susceptibility was measured using susceptibility bridge, part of Molspin Minispin designed by L. Molyneux.

\section{Results and discussion}

\subsection{Effect of $Z n$ addition on the magnetic susceptibility and magnetization of $\mathrm{CoFe}_{2} \mathrm{O}_{4}$ ferrites}

Figures 1 and 2 present graphs of magnetic susceptibility $(\chi)$ and the magnetization $(M)$ as iunctions of $x$.

The increase of $\chi$ and $M$ with $\mathrm{Zn}$ addition $x$ is discussed as follows. The mixed cobalt-zinc ferrites have the formula $\mathrm{Zn}_{x}^{\prime \prime} \mathrm{Fe}_{1-x}^{\prime \prime \prime}\left(\mathrm{Me}_{1-x}^{\prime} \mathrm{Fe}_{1+x}^{\prime \prime \prime}\right) \mathrm{O}_{4}$ with $\mathrm{Me}$ as the magnetic ion $\mathrm{Co}^{2+}, \mathrm{Zn}^{2+}$ is known to have a preferential occupations for the tetrahedral A-sites. The presence of cobalt on the octahedral sites of the spinel
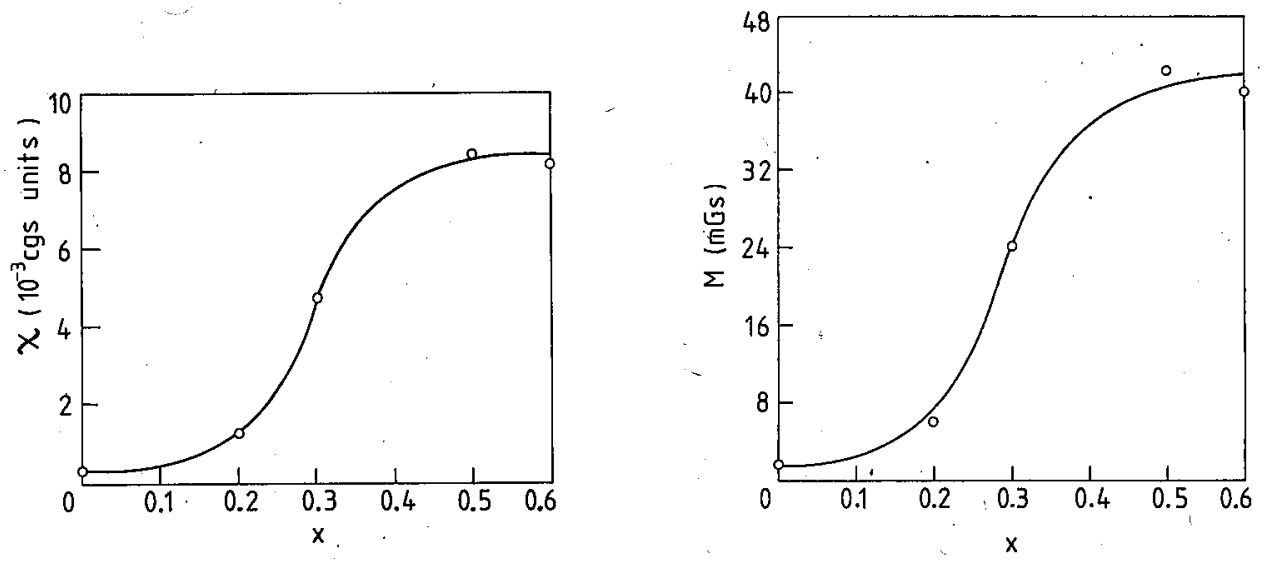

Fig. 1. Variation of magnetic susceptibility $\chi$ with the zinc addition $x$.

Fig. 2. Variation of magnetization $M$ with the zinc addition $x$.

favours a conduction mechanism $\mathrm{Co}^{2+}+\mathrm{Fe}^{3+} \rightleftharpoons \mathrm{Co}^{3+}+\mathrm{Fe}^{2+}$ which explains the predominant conduction mechanism in $\mathrm{CoZnFe}_{2} \mathrm{O}_{4}$. The substituting zinc ions instead of cobalt ions in the composition.

Cobalt ferrite $\left(\mathrm{CoFe}_{2} \mathrm{O}_{4}\right)$ has the inverse spinel structure in which the tetrahedral sites are occupied by $\mathrm{Fe}^{3+}$ and the octahedral sites are occupied half by $\mathrm{Fe}^{3+}$ and half by $\mathrm{Co}^{2+}$. The excess cobalt or iron occupies the tetrahedral sites as $\mathrm{Co}^{3+}$ or $\mathrm{Fe}^{2+}[6]$ then generates holes or electrons as

$$
\begin{aligned}
& \mathrm{Co}^{3+} \rightarrow \mathrm{Co}^{2+}+\mathrm{h}^{+}, \\
& \mathrm{Fe}^{2+} \rightarrow \mathrm{Fe}^{3+}+\mathrm{e}^{-} .
\end{aligned}
$$


The increase of $\mathrm{Zn}$ addition caused the movement of $\mathrm{Fe}^{3+}$ ions from the tetrahedral to octahedral sites. This should means an initial increase in the resultant magnetization on the B-sites.

\subsection{Magnetization dependence on lattice parameter a}

The lattice parameter of $\mathrm{CoFe}_{2} \mathrm{O}_{4}$ is determined to be $0.8385 \mathrm{~nm}$, which is compared favourably with the value of $0.8394 \mathrm{~nm}$ [5]. The variation of lattice parameter $a$ as a function of zinc addition $x$ in $\mathrm{Co}_{1-x} \mathrm{Zn}_{x} \mathrm{Fe}_{2} \mathrm{O}_{4}$ is previously represented. It showed that the lattice parameter increase linearly with the increase of zinc content.

The X-ray studies showed that the materials were in all cases homogeneous having the spinel structure.

We have for the magnetizations $M_{\mathrm{A}}$ and $M_{\mathrm{B}}$ of the $\mathrm{A}$ - and B-lattice sites, respectively, for $N$ magnetic ions (only one kind) [4]:

$$
\begin{gathered}
M_{\mathrm{A}}=\lambda N g \beta S B_{\mathrm{s}}\left(g \beta S H_{\mathrm{A}} / k T\right), \\
M_{\mathrm{B}}=\mu N g \beta S B_{\mathrm{s}}\left(g \beta S H_{\mathrm{B}} / k T\right),
\end{gathered}
$$

where $\lambda$ and $\mu$ are the fraction of magnetic ions on the A- and B-sites, respectively; $H_{\mathrm{A}}$ and $H_{\mathrm{B}}$ are the effective field acting on the magnetic ions in the $\mathrm{A}$ - and $\mathrm{B}$-sites, respectively, which are expressed in terms of the molecular field coefficients ( $\gamma$ 's) as

$$
\begin{aligned}
H_{\mathrm{A}} & =H_{0}+\gamma_{\mathrm{AA}} M_{\mathrm{A}}-\gamma_{\mathrm{AB}} M_{\mathrm{B}}, \\
H_{\mathrm{B}} & =H_{0}-\gamma_{\mathrm{AB}} M_{\mathrm{A}}+\gamma_{\mathrm{BB}} M_{\mathrm{B}},
\end{aligned}
$$

where $\gamma_{i j}$ is the Weiss molecular field coefficient for the interaction between the ions on the $i_{\text {th }}$ and $j_{\text {th }}$ sites, $B_{\mathbf{s}}()$ is the Brillouin function.

In the ferrimagnetic case we obtain the spontaneous magnetization $\left(H_{0}=0\right)$ from Eqs. (1) and (2) in terms of the specific magnetization $\mathcal{Y}_{\mathrm{A}}$ and $\mathcal{Y}_{\mathrm{B}}$, given in terms of the number of Bohr magnetons per molecule as

$$
\begin{array}{r}
\mathcal{Y}_{\mathrm{A}}=B_{\mathrm{s}}\left[(\tau / \mathrm{T})\left(\lambda \gamma_{\mathrm{AA}} \mathcal{Y}_{\mathrm{A}}-\mu \gamma_{\mathrm{AB}} \mathcal{Y}_{\mathrm{B}}\right)\right] \\
\mathcal{Y}_{\mathrm{B}}=B_{\mathrm{s}}\left[(\tau / \mathrm{T})\left(-\lambda \gamma_{\mathrm{AB}} \mathcal{Y}_{\mathrm{A}}+\mu \gamma_{\mathrm{BB}} \mathcal{Y}_{\mathrm{B}}\right)\right]
\end{array}
$$

where $\tau=N g^{2} \beta^{2} s^{2} / k$, with

$$
\mathcal{Y}_{\mathrm{A}}=M_{\mathrm{A}} / \lambda N g \beta s, \quad \mathcal{Y}_{\mathrm{B}}=N_{\mathrm{B}} / \mu N g \beta S
$$

The net specific magntization $\mathcal{Y}$ is given by

$$
\mathcal{Y}=\left|\lambda \mathcal{Y}_{\mathrm{A}}+\mu \mathcal{Y}_{\mathrm{B}}\right|
$$


From this theory Néel has calculated $\mathcal{Y}$ as a function of the temperature and arranged the curves into various types according to their shapes. The classification is made in terms of the parameters $\alpha$ and $\beta$ which are defined as follows:

$$
\alpha=\gamma_{\mathrm{AA}} /\left|\gamma_{\mathrm{AB}}\right|, \quad \beta=\gamma_{\mathrm{BB}} /\left|\gamma_{\mathrm{AB}}\right| .
$$

The $\alpha$ 's and $\beta$ 's give the strength and sign of the AA and BB interactions, respectively, in terms of the AB interaction.

In the present case, instead of one type of magnetic ion, as developed by Néel, we have two of them which can occupy either or both the A- or B-sites; and, furthermore, one cannot assume that the $g$ factor for a given magnetic ion will be the same in both the A- and B-sites. This imposes mathematical complexities,
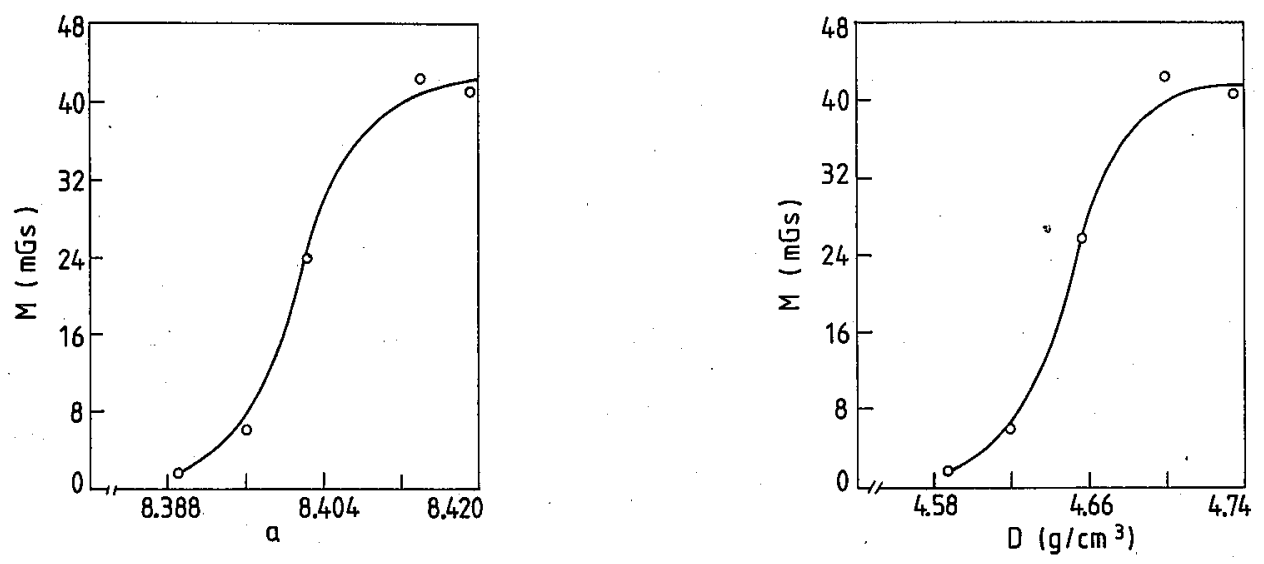

Fig. 3. Variation of magnetization $M$ with the lattice parameter $a$.

Fig. 4. Variation of magnetization $M$ with the bulk density $D$.

for Eqs. (1) through (4) would have to be modified accordingly before a detailed comparison with the theory could be accomplished. However, certain qualitative conclusions can be made.

For greater amounts of zinc and increase of the crystal size (Fig. 3), the magnetization from the B-sites predominates and the coupling weakens at the A-sites.

In conclusion, the $\mathrm{Co}_{1-x} \mathrm{Zn}_{x} \mathrm{Fe}_{2} \mathrm{O}_{4}$ system can be prepared with a systematic linear variation in size of the unit cell which caused the magnetic strength of the magnetic ions on the $\mathrm{A}$ - and $\mathrm{B}$-sites to vary relative to each other by the substitution of $\mathrm{Zn}^{2+}$ with a situation arising in the case of cobalt-zinc ferrite, where the magnetization on the B- and A-sites is not equal and opposite. 


\subsection{Effect of density on the magnetization of $\mathrm{Co}_{1-x} \mathrm{Zn}_{x} \mathrm{Fe}_{2} \mathrm{O}_{4}$ ferrites}

The effect of density on the magnetization $(M)$ and the magnetic susceptibility $(\chi)$ of $\mathrm{Co}_{1-x} \mathrm{Zn}_{x} \mathrm{Fe}_{2} \mathrm{O}_{4}$ are shown in Figs. 4 and 5. The increase of $M$ and $\chi$

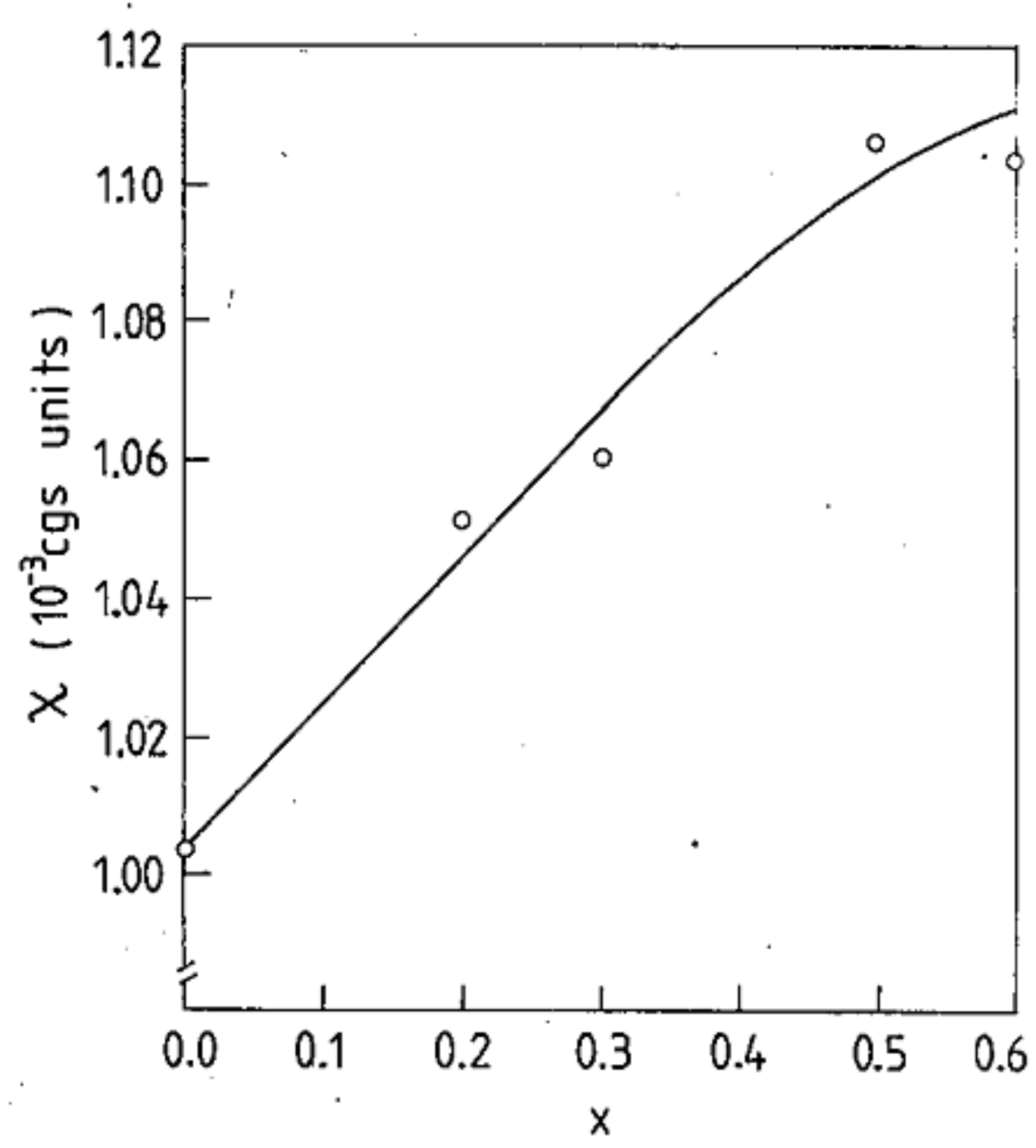

Fig. 5. Variation of magnetic susceptibility $\chi$ with the addition of $\mathrm{Zn} x$.

with density is due to the following: The increase of density reduced the porosity of the composition giving rise to the increase of the magnetic interaction at the B-sites. This interaction increases $M$ with increasing density.

\section{Acknowledgement}

Measurements of the magnetic susceptibility were carried out at Paleomagnetic Laboratory Polytechnic Southwest, Plymouth, Devon, United Kingdom.

The X-ray diffraction and other measurements were performed at the Center Laboratory of Monofia University and Solid State Phys. Laboratory, Faculty of Science, Tanta University.

\section{References}

[1] A. Michel, E. Pouillard, C. R. Hebd. Seances Acad. Sci. 227, 194 (1948).

[2] L.R. Maxwell, J.S. Smart, S. Brunauer, J. Chem. Phys. 19, 303 (1951).

[3] C. Guillaud, A. Michel, J. Phys. et Radium 12, 65 (1951).

[4] L. Néel, Ann. Phys. (U.S.A.) 3, 137 (1948).

[5] M.A. Ahmed, Phys. Status Solidi A 111, 567 (1989).

[6] Y. Yamazaki, M. Satdu, Jpn. J. Appl. Phys. 12, 998 (1973). 